\title{
Encefalitis anti NMDAR: Reporte de caso con seguimiento a largo plazo
}

\author{
Anti-NMDAR Encephalitis: Case report with a long - term follow-up \\ Miguel A. Vences ${ }^{1, a}$, Víctor V. Saquisela ${ }^{1, b}$, Elliot Barreto-Acevedo ${ }^{1, b}$, Marco A. Zuñiga ${ }^{1 b}$ \\ RESUMEN
}

La encefalitis autoinmune por anticuerpos antineurales de superficie, abarca un amplio espectro de entidades clínicas. La encefalitis por anticuerpos contra el antígeno de superficie de la porción externa del receptor del N-metil-D-aspartato (RNMDA) es la más frecuente y de mejor caracterización. Se reporta el caso de un adolescente con un cuadro clínico neurosiquiátrico y crisis epilépticas de reciente inicio, que presentó respuesta positiva para anticuerpos anti-RNMDA y respuesta parcial a tratamiento con corticoterapia e inmunoglobulina; en vista de esto, recibió manejo adicional con recambio plasmático seguido por terapia de mantenimiento con ciclos de inmunoglobulina, sin uso de inmunosupresores. Se reportan los resultados del seguimiento a largo plazo.

PALABRAS CLAVE: Encefalitis; N-Metilaspartato; resultado del tratamiento.

\section{SUMMARY}

Autoimmune encephalitis due to surface antineural antibodies covers a wide spectrum of clinical entities. Encephalitis due to antibodies against the surface antigen of the external portion of the N-methyl-D-aspartate receptor (RNMDA) is the most frequent and best characterized. The case of an adolescent with a clinical picture of neurosychiatric disorder and epileptic seizures of recent onset is presented: he had a positive response for anti-RNMDA antibodies, and a partial response to treatment with corticosteroid therapy and immunoglobulin; therefore, he received additional management with plasma exchange followed by maintenance therapy with immunoglobulin cycles, without the use of immunosuppressants. The results of a long-term follow-up are reported.

KEYWORDS: Encephalitis; N-Methylaspartate ; treatment outcome.

\section{INTRODUCCIÓN}

La encefalitis autoinmune por anticuerpos antineurales de superficie, es una enfermedad poco frecuente, descrita como tal por primera vez por Joseph Dalmau en el año 2007 y comprende un amplio espectro de entidades clínicas (1). La etiopatogenia está relacionada a anticuerpos antineurales que se dirigen contra antígenos de superficie; el anticuerpo frente a la porción externa del receptor del N-metil-Daspartato (RNMDA), es el más frecuente y principal responsable de las encefalitis de causa indeterminada (2).

Actualmente, su diagnóstico sigue siendo un reto debido a la variabilidad clínica de presentación, el patrón temporal subagudo y la baja tasa de sospecha clínica al ingreso; siendo muchos de los pacientes internados en centros de salud mental, con diagnósticos errados de enfermedades psiquiátricas primarias (3). Existe con frecuencia un retardo en el diagnóstico y en países como el nuestro, la ausencia de la prueba

1 Departamento de Neurología, Servicio de Epilepsia y Enfermedades Cerebrovasculares, Hospital Nacional Edgardo Rebagliati Martins, Essalud. Lima. Perú.

a Médico residente de Neurología; ${ }^{b}$ Médico Neurólogo Asistente 
confirmatoria en hospitales públicos dificulta la tarea diagnostica del especialista; por lo que es importante conocer acerca de esta enfermedad, para realizar un tratamiento temprano ya que esto influirá en el pronóstico de los pacientes afectados (4).

Se reporta el caso clínico de un paciente con encefalitis por anticuerpos contra RNMDA y su seguimiento al año de evolución, con la finalidad de realizar una revisión de la literatura enfocada en la conducta terapéutica y de seguimiento.

\section{Presentación del caso}

Paciente varón de 17 años, sin antecedentes patológicos, inició cuadro clínico subagudo de instalación en cinco días, caracterizado por cefalea global intensa, alucinaciones auditivas simples, visuales complejas, risa inmotivada y un evento de crisis tónico-clónico generalizada de inicio desconocido durante el sueño. Posteriormente se añadió fiebre, alteración conductual, disforia y agresividad; además presentó ecolalia y ecopraxia, por lo que fue ingresado por emergencia de Salud Mental de nuestro hospital, bajo la sospecha de trastorno delirante orgánico.

Durante su hospitalización en psiquiatría cursó con fiebre persistente, se le realizó punción lumbar con resultado del citoquímico del líquido cefalorraquídeo (LCR) normal. Se inició tratamiento empírico con aciclovir endovenoso (EV) y Carbamazepina $600 \mathrm{mg} /$ día. En la semana siguiente la evolución fue tórpida, añadiéndose mutismo, discinecias orofaciales y recurrencia de crisis tónico-clónicas generalizadas. Por ello fue transferido a hospitalización de neurología de nuestro hospital. A su ingreso al servicio el paciente se encontraba, despierto, inatento, con mutismo acinético, discinesias orofaciales, movimientos coreo atetósicos en extremidades y respuesta plantar extensora bilateral sin meningismo. Se realizó una resonancia magnética sin hallazgos significativos, un electroencefalograma que evidenció desorganización y lentificación difusa, ocasionales ondas delta polimorfas de morfología aguda con distribución fronto - temporal derecha, sin actividad epileptiforme (figura 1).

Debido a las características semiológicas del cuadro clínico, el patrón temporal subagudo, la normalidad del citoquímico del LCR y la pobre respuesta del paciente al aciclovir (escala de Rankin modificada de 5 puntos), se replanteó el diagnóstico de encefalitis autoinmune probable; se realizó un amplio diagnóstico diferencial con resultados séricos normales para enfermedades autoinmunes sistémicas e infecciosas, incluido la quioluminiscencia para el virus VIH. Además se

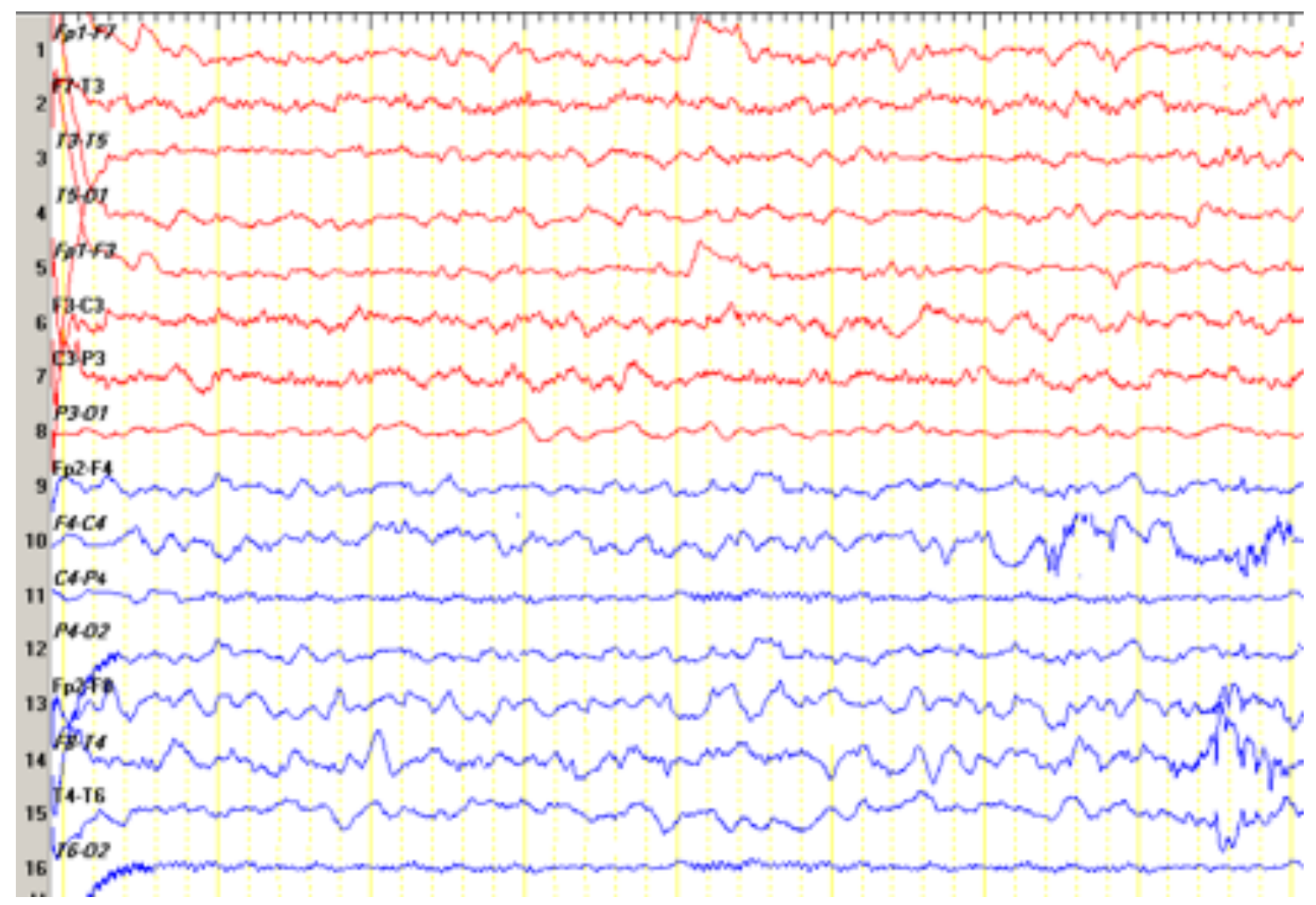

Figura 1. Electroencefalograma: Lentificación difusa continua con ondas delta de morfología aguda de distribución fronto - temporal derecha intermitente, sin actividad epileptiforme. 


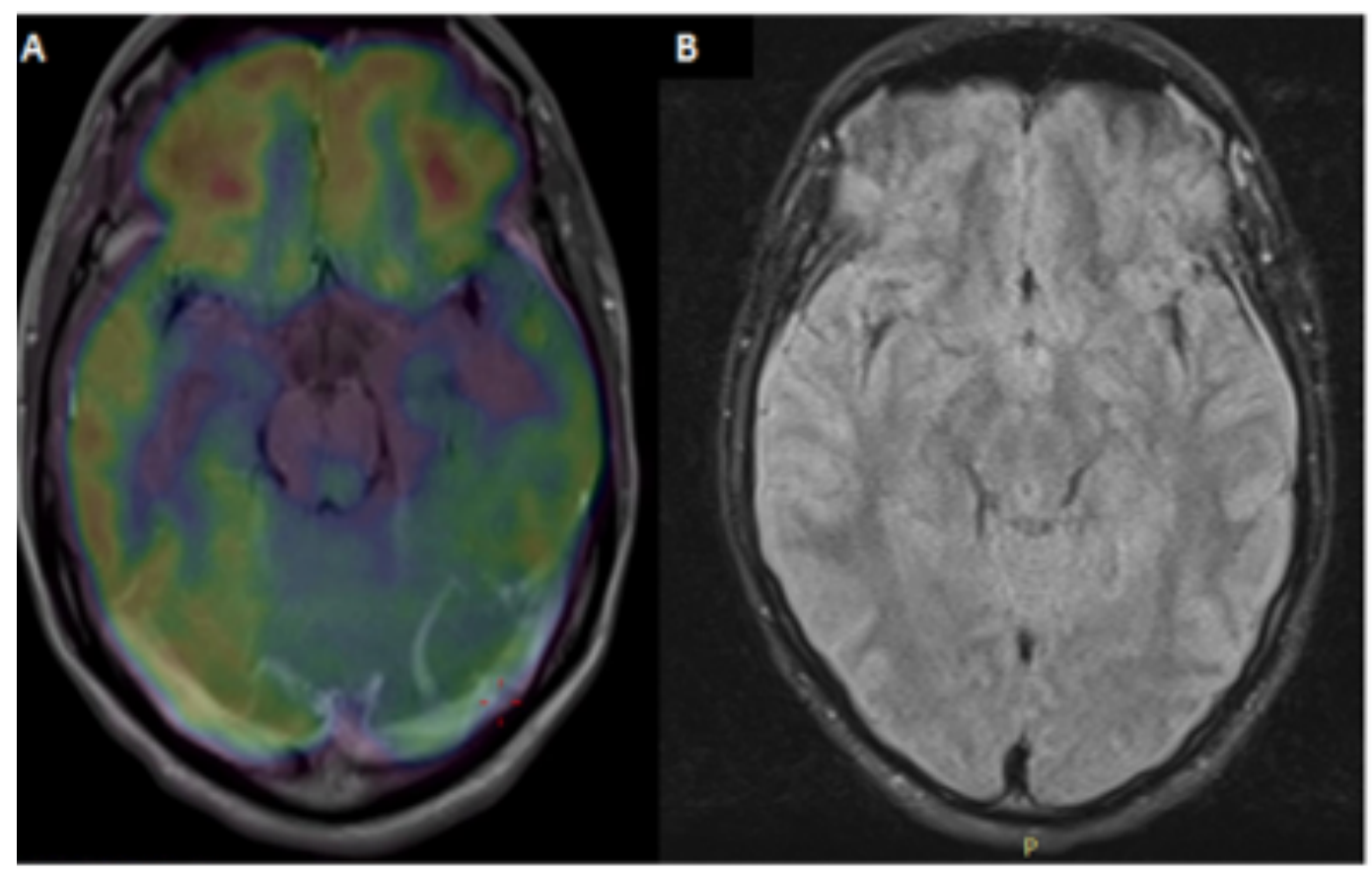

Figura 2. A) PET SCAN con FDG-18.Hipometabolismo en corteza dorsolateral de lóbulo occipital izquierdo e hipermetabolismo en corteza orbitofrontal bilateral de predominio izquierdo. B) Resonancia magnética de encéfalo. Secuencia FLAIR, sin hallazgos significativos.

realizó una nueva punción lumbar para análisis de anticuerpos Ig G contra la subunidad NR1 del receptor NMDA, utilizando el método de cultivo de células transfectadas, siendo el resultado citoquímico normal. Se realizó una tomografía cervical, torácica, abdominal y pélvica con contraste que excluyó presencia de neoplasias y un estudio de tomografía por emisión de positrones (PET SCAN) corporal total con Fluorodeoxiglucosa 18 (FDG-18) (figura 2), evidenciándose en el encéfalo un hipometabolismo en corteza dorsolateral de lóbulo occipital izquierdo e hipermetabolismo en corteza orbitofrontal bilateral de predominio izquierdo, sin evidencia de lesiones o áreas focales de metabolismo anormal que sugiriera actividad neoplásica en el resto del estudio.

A las dos semanas de iniciado el cuadro clínico, se inició tratamiento inmunosupresor con pulsos de un gramo de metilprednisolona EV por cinco días, sin respuesta clínica. Entonces se inició tratamiento inmunomodulador con inmunoglobulina a 2g/ $\mathrm{Kg}$ dividido en cinco días, con mejoría parcial en la atención, la conducta y el lenguaje (respondía monosilábicamente algunas preguntas simples). Luego de dos semanas, debido a que solo se obtuvo mejoría modesta; se añadió tratamiento con recambio plasmático total en cinco sesiones.
A la semana de iniciado el recambio plasmático total, el paciente logró una mejora clínica evidente del lenguaje con un dialogo fluido, comprensión y ejecución de actividades (escala de Rankin modificada de 1 punto) y sin trastorno conductual; se recibió el resultado de anticuerpos contra receptores NMDA siendo positivo con un título de 1/64. Es dado de alta a las seis semanas de enfermedad, sin recurrencia de crisis epilépticas y con un logro total de independencia funcional; con una escala anti-NMDAR Encephalitis One-Year Functional Status (NEOS) de 1 punto. Se decidió que el paciente reciba ciclos de mantenimiento mensuales con inmunoglobulina EV a $1,2 \mathrm{gr} / \mathrm{Kg}$ dividido en tres días, por seis meses y continuó con fármacos antiepilépticos.

Actualmente al año del evento, habiendo completado seis ciclos de mantenimiento con Inmunoglobulina, el paciente se mantiene con buena evolución encontrándose al examen clínico: Despierto, atento, sin déficit motor, autovalente (escala de Rankin modificada de 0 ), con regular desempeño académico universitario, sin crisis epilépticas, con una Evaluación Cognitiva de Montreal (MoCA) de 29 puntos; y sin recurrencia del cuadro de encefalitis autoinmune hasta la fecha. 


\section{DISCUSIÓN}

La encefalitis por anticuerpos contra RNMDA es la más frecuente entre las encefalitis autoinmunes por anticuerpos antineurales de superficie y se genera por la respuesta inmunomediada frente a la porción externa del receptor del NMDA, con ello se genera un desequilibrio en la transmisión sináptica y de mayor repercusión a nivel hipocampal donde estos receptores están presentes en mayor proporción (5). Se ha postulado en diferentes estudios que estos mecanismos se generarían luego de la exposición a una infección viral o a células neoplásicas, lo que explicaría por qué en muchos de estos pacientes se haya reportado una sobreposición a estas afecciones (6). Es más frecuente en mujeres y personas jóvenes, además está relacionada en poco más de la mitad de los casos a neoplasias ocultas, siendo la más frecuente reportada el teratoma ovárico y el factor de riesgo más importante para esta asociación es la edad de presentación más temprana $(1,6)$.

La ausencia de la prueba confirmatoria (panel para encefalitis autoinmune) en la mayoría de hospitales públicos en países en vías de desarrollo, hace difícil determinar cuál es la verdadera prevalencia de esta enfermedad. En Latinoamérica, las series de casos más grandes son de 11 pacientes en Argentina (7) y 13 en Chile (8); en contraste a series extranjeras como el estudio multicéntrico realizado en Inglaterra por Granerod (2) y la cohorte del Proyecto California (9), donde se reportó como la causa del $21 \%$ y $41 \%$ del total de encefalitis, respectivamente. Asimismo la negatividad de la prueba confirmatoria, en un paciente con cuadro clínico sugestivo y de etiología aún no determinada, no excluye que el desorden sea inmunomediado. Debido al reto diagnóstico y la demora en el procesamiento de la prueba confirmatoria se han diseñado criterios diagnósticos de probabilidad de encefalitis anti NMDAR, con el objetivo de no diferir el tratamiento oportuno para estos pacientes (10).

El cuadro clínico de la encefalitis autoinmune, está caracterizado por la presencia prodrómica de cefalea precoz, seguida del inicio temprano de síntomas corticales (alteración del comportamiento, crisis epilépticas, déficit en la cognición) y presentación tardía de síntomas subcorticales (trastornos del movimiento, disfunción autonómica) (1). En el caso de nuestro paciente dichas manifestaciones clínicas se presentaron de forma subaguda con un predominio en la alteración conductual, lo cual es característico de este tipo de encefalitis. Las crisis epilépticas tónico
- clónico generalizadas, son las más frecuentemente reportadas, siendo la fármaco sensibilidad algo que las permite distinguir de otro tipo de encefalitis autoinmune tales como las causadas por anticuerpos contra el receptor GABA-A (11). Además el riesgo de desarrollo de epilepsia crónica en las encefalitis autoinmunes tratadas de manera exitosa es menor al $15 \%$ de los casos (12).

Respecto a los exámenes paraclínicos, la resonancia magnética es normal para más de la mitad de pacientes, como en nuestro caso. En el examen citoquímico del LCR, se describe que la mayoría de casos presentan pleocitosis linfocitaria, y la elevada celularidad $(>20$ células/ uL), sería un predictor independiente de un pobre estado funcional al año de evolución (4). En nuestro caso la celularidad del LCR fue normal y su evolución funcional fue buena. La actividad delta fronto-temporal derecha evidenciada en el EEG del paciente, da cuenta de una disfunción focal anterior, esperable en este tipo de encefalitis. Sin embargo, no registramos actividad epileptiforme ni el patrón delta brush. Este patrón ha sido descrito como típico de los pacientes afectados por NMDAR, pero que solo está presente en una tercera parte de ellos, además es un factor asociado a epilepsia farmacorresistente y deterioro de nivel de conciencia con necesidad de ingreso a unidad de cuidados críticos $(1,4)$.

Los estudios de barrido como la tomografía y PET SCAN están justificados en búsqueda de neoplasias ocultas asociadas a esta entidad; tal es el caso de la asociación en pacientes mujeres en edad reproductiva con teratomas ováricos y en el sexo masculino la asociación esporádica a carcinomas de pulmón de células pequeñas, teratoma mediastinal y linfoma (5). Además se ha reportado la utilidad del PET SCAN de encéfalo en el diagnóstico inicial cuando la resonancia magnética es negativa, en especial en los casos de mayor severidad. El patrón más frecuentemente descrito es la presencia de hipermetabolismo orbitofrontal y temporal asociado a hipometabolismo parieto-occipital, a lo que se ha denominado gradiente frontotemporal-occipital (13). Este patrón descrito fue observado en el PET SCAN que se realizó al paciente. Sin embargo, los resultados sobre el patrón descrito en PET cerebral no se han evidenciado en todos los casos, por lo que su valor diagnostico no es concluyente y se ha planteado un mayor valor en el seguimiento (14).

El esquema terapéutico de la encefalitis autoinmune es determinado en base a recomendación de expertos; la mayoría concuerda en un primer ciclo 
de tratamiento inmunosupresor o inmunomodulador con pulsos de metilprednisolona ( $1 \mathrm{~g} /$ día por 5 días), Inmunoglobulina Humana intravenosa $(0,4 \mathrm{~g} / \mathrm{Kg} /$ día por 5 días) o plasmaféresis. Si no hay respuesta clínica a las 2 semanas o es limitada luego del primer ciclo de inmunoterapia, se considera un segundo ciclo con terapia de segunda línea: Ciclofosfamida (750 mg/m2) o Rituximab $(375 \mathrm{mg} / \mathrm{m} 2$, semanal por 4 semanas). Luego de ello, para evitar recaídas que pueden presentarse hasta en el $12 \%$ de los pacientes (15), se recomienda terapia de mantenimiento con micofenolato o azatioprina por al menos un año; respecto al uso de Inmunoglobulina no se ha establecido una régimen estándar, la mayoría de pacientes han recibido dosis de mantenimiento cada mes por un tiempo variable (16). En nuestro paciente se utilizó un esquema combinado de primer ciclo con metilprednisolona asociado a Inmunoglobulina. Sin embargo al considerarse que ya había transcurrido 4 semanas de iniciado el cuadro clínico sin mejoría concluyente (lo que podría conllevar un peor pronóstico funcional y cognitivo a largo plazo), se decidió realizar un ciclo de recambios plasmáticos sin esperar el tiempo de 2 semanas de terminado el ciclo de Inmunoglobulina. La respuesta clínica fue favorable; lo que llevaría a plantear que en este tipo de patologías quizás se logra una respuesta más pronta con la plasmaféresis respecto a la Inmunoglobulina, tal y como sucede en otras patologías, sin significar ello que una opción sea más efectiva que la otra.

A la fecha no se ha reportado en forma concluyente algún predictor de la respuesta a la inmunoterapia. Algunos estudios la han correlacionado a una mayor concentración de albumina sérica $(>4 \mathrm{mg} / \mathrm{dL})$ como un factor de buena respuesta a la Inmunoglobulina (17). En el caso de nuestro paciente tenía un nivel de albúmina sérico de 4,6 mg/dl, el cual se correlacionó con respuesta parcial a dicha terapia a la semana.

La escala NEOS, predice la función neurológica 1 año después del diagnóstico de Encefalitis anti NMDAR, y considera las siguientes variables (18): Ingreso a la unidad de cuidados intensivos, retraso del tratamiento $>4$ semanas, falta de mejora clínica dentro de 4 semanas, resonancia magnética anormal y recuento de glóbulos blancos en el LCR $>20$ células / $\mu \mathrm{L}$. Tiene una correlación directa con pobre resultado funcional (escala de Rankin Modificada >2); en nuestro paciente la puntuación fue de un punto, lo que predecía la buena funcionalidad al año de evolución lo que se corroboró posteriormente.
Debido al compromiso perihipocampal comprometido en estos pacientes, no solo existe la necesidad de evaluar la funcionalidad del paciente, sino también los resultados cognitivos a largo plazo; siendo la evaluación Cognitiva de Montreal (MoCA), una herramienta útil en estos pacientes. El estudio de Julien Hébert, asoció un retraso menor a 60 días en el tratamiento y la ausencia de estado epiléptico al inicio, con un mejor rendimiento cognitivo en esta escala (19). En nuestro caso no hubo deterioro cognitivo, con un puntaje de 29 puntos, el cual se correlaciona con los hallazgos obtenidos en estos pacientes.

En conclusión, la encefalitis autoinmune anti NMDAR es una entidad sub-diagnosticada que siempre se debe tener presente en el contexto clínico adecuado; se debe de realizar un diagnóstico y tratamiento oportuno ya que es un factor predictor de buen pronóstico tanto funcional como cognitivo.

\section{Correspondencia:}

Dr. Miguel Angel Vences Mijahuanca

Hospital Nacional Edgardo Rebagliati Martins, Departamento de Neurología

Av. Rebagliati 490 Jesús María. Lima, Perú

Correo electrónico: vens1793@hotmail.com

ORCID ID: https://orcid.org/0000-0002-8538-6242

\section{REFERENCIAS BIBLIOGRÁFICAS}

1. Dalmau J, Gleichman A, Hughes E, et al. Anti-NMDAreceptor encephalitis: case series and analysis of the effects of antibodies. Lancet Neurol. 2008; 7(12):1091-8. doi: 10.1016/S1474-4422(08)70224-2

2. Granerod J, Ambrose H, Davies N, et al. Causes of encephalitis and differences in their clinical presentations in England: a multicentre, population-based prospective study. Lancet Infect Dis. 2010;10(12):835-44. doi: 10.1016/S14733099(10)70222-X

3. Custodio N, Escobar-Montalvo J. Neurophychiatric symptoms in anti-N-methyl-D-aspartate receptor encephalitis: apropos of two cases. An Fac med. 2016; 77(3):277-81.

4. Titulaer M, McCracken L, Gabilondo I, et al. Treatment and prognostic factors for longterm outcome in patients with anti-NMDA receptor encephalitis: an observational cohort study. Lancet Neurol 2013; 12:157-165.

5. Sansing L, Tüzün E, Ko M, Baccon J, Lynch D, Dalmau J. A patient with encephalitis associated with NMDA receptor antibodies. Nat Clin Pract Neurol. 2007; 3(5):291-6. 
6. Dalmau J, Lancaster E, Martinez-Hernandez E, Rosenfeld M, Balice-Gordon R. Clinical experience and laboratory investigations in patients with antiNMDAR encephalitis. Lancet Neurol. 2011; 10: 6374.

7. Péres E, Ruggieri V, Monges S, et al. Encefalitis mediada por anticuerpos contra el receptor ionótropico de Glutamato activado por N-metil-d-aspartato: análisis de once casos pediátricos en Argentina. Medicina. 2013; 73 (1): 1-9.

8. Erazo R, Gonzales J, Quintanilla C. Encefalitis subaguda por anticuerpos anti receptor de N-metil-Daspartato. Serie de 13 casos pediátricos. Rev Chil Pediatr. 2016; 87(6): 487-493.

9. Gable M, Sheriff H, Dalmau J, Tilley D, Glaser C. The frequency of autoimmune N-methyl-D-aspartate receptor encephalitis surpasses that of individual viral etiologies in young individuals enrolled in the California encephalitis project. Clin Infect Dis. 2012; 54 (7): 899-904.

10. Graus F, Titulaer M, Balu R, et al. A clinical approach to diagnosis of autoimmune encephalitis. Lancet Neurol. 2016;15(4):391-404. doi: 10.1016/S14744422(15)00401-9

11. Dubey D, Samudra N, Gupta P, et al. Retrospective case series of the clinical features, management and outcomes of patients with autoimmune epilepsy. Seizure. 2015; 29: 143-7.

12. Spatola M, Dalmau J. Seizures and risk of epilepsy in autoimmune and other inflammatory encephalitis. Curr Opin Neurol. 2017; 30(3):345-353. doi: 10.1097/ WCO.0000000000000449
13. Heine J. Imaging of autoimmune encephalitis Relevance for clinical practice and hippocampal function. Neuroscience. 2015; 309: 68-83.

14. Morbelli S, Arbizu J, Booij J, et al. The need of standardization and of large clinical studies in an emerging indication of $[18 \mathrm{~F}]$ FDG PET: the autoimmune encephalitis. Eur J Nucl Med Mol Imaging. 2017; 44(3):353-357.

15. Collao J, Romero C, Delgado C. Encefalitis autoinmunes: criterios diagnósticos y pautas terapéuticas. Rev Med Chile. 2018; 146: 351-361.

16. Rosenfeld M, Dalmau J. Anti-NMDA-Receptor Encephalitis and Other Synaptic Autoimmune Disorders. Curr Treat Options Neurol. 2011; 13 (3): 324-32.

17. Jang Y, Lee ST, Kim TJ, et al. High albumin level is a predictor of favorable response to immunotherapy in autoimmune encephalitis. Scientific Reports. 2018; 8: 1012.

18. Balu R, McCracken L, Lancaster E, Graus F, Dalmau J, Titulaer MJ. A score that predicts 1-year functional status in patients with anti-NMDA receptor encephalitis. Neurology. 2019;92(3):e244-e252. doi: 10.1212/WNL.0000000000006783

19. Hébert J, Day G, Steriade C, Wennberg R, TangWai D. Long-Term cognitive outcomes in patients with autoimmune encephalitis. Can J Neurol Sci. 2018;45(5):540-544.

Recibido: 09/02/2020

Aceptado: 02/06/2020 